\title{
Invertible Minkowski Sum of Polygons
}

\author{
Kokichi Sugihara \\ Department of Mathematical Informatics, University of Tokyo \\ Hongo, Bunkyo-ku, Tokyo 113-0033, Japan \\ sugihara@simplex.t.u-tokyo.ac.jp
}

\begin{abstract}
The paper gives a new formulation of the Minkowski sum of polygons. In the conventional Minkowski sum, the inverse operation is not well defined unless the polygons are restricted to be convex. In the proposed formulation, on the other hand, the set of polygons is extended to the set of "hyperpolygons" and the Minkowski sum forms a commutative group. Consequently, every polygon has its unique inverse, and the sum and the inverse operations can be taken freely. An example of a physical interpretation of the hyperpolygon is also given.
\end{abstract}

\section{Introduction}

The Minkowski sum is one of the most fundamental operations for geometric objects, and has a wide range of applications in data compression [4, generation of collision-free paths [10, and others. The Minkowski sum is also useful for constructing efficient geometric algorithms [6].

Algorithms for computing the Minkowski sum have also been studied extensively; they include algorithms for restricted classes of polygons 813, for general polygons 612, and for higher dimensional figures 149.

However, there is a big open problem on the "inverse" of the Minkowski sum, because no clear definition of the inverse is known yet for a general class of figures. An inverse-like operation called the Minkowski difference is known, but it behaves as the inverse only for strongly restricted cases such as the case of convex figures [3. Guibas et al. 6] studied objects which are obtained by the inverselike operation and pointed out that the boundaries of those objects change their directions partially. Ghosh 34 tried to define the inverse for nonconvex figures, but his definition is valid for a certain restricted class only.

Sugihara et al. 14 solved this problem partially. They introduced a class of closed curves whose tangent direction is continuous and monotone, and defined an algebraic operation in this class. This operation is a generalization of the Minkowski sum in that if we replace these closed curves with the regions bounded by the curves, the operation coincides with the Minkowski sum. Moreover, the operation is invertible in this class, and thus the inverse of the Minkowski sum is defined for a much wider class than the class of convex figures.

In this paper, we apply a similar idea to polygons, and construct an algebraic system in which a generalization of the Minkowski sum and its inverse is well defined. In this new system, the set of polygons is extended to a set of more

A. Braquelaire, J.-O. Lachaud, and A. Vialard (Eds.): DGCI 2002, LNCS 2301, pp. 350-359 2002. (C) Springer-Verlag Berlin Heidelberg 2002 
general objects, which we call "hyperpolygons". The extension from polygons to hyperpolygons has the same structure as the extension from integers to rationals.

After we review conventional Minkowski algebra in Section 2, we introduce a new representation of the boundary of polygons in Section 3. Next, we define the new Minkowski sum on this representation in Section 4, and extend it to a larger invertible world in Section 5. In Section 6, we give an example of an interpretation of a physical meaning of a hyperpolygon, and make some concluding remarks in Section 7.

\section{Conventional Minkowski Sum}

We fix an $(x, y)$ Cartesian coordinate system $\Sigma$, and represent a point by its radial vector with respect to the origin of $\Sigma$. We call a set of points a figure. For two figures $A$ and $B$, the new figure defined by

$$
A \oplus B=\{a+b \mid a \in A, b \in B\}
$$

is called the Minkowski sum of $A$ and $B$, where $a+b$ represents the sum of the radial vectors $a$ and $b$.

Let $A$ and $B$ be the convex figures in Fig. 1(a) and (b). Then, the Minkowski sum of $A$ and $B$ is the figure $C$ shown in Fig. 1(c).

The Minkowski sum can be intuitively understood in the following way. Consider the figures $A$ and $B$ in Fig. 1(a) and (b) again. Let us choose and fix an arbitrary point in $B$, as the dotted point in Fig. 1(b), and call it the reference point. As shown in Fig. 1(d), the Minkowski sum is the union of the figure $A$ and the region swept by $B$ when the reference point of $B$ moves along the boundary of $A$.

Next suppose that we are given two figures $B$ and $C$. We want to find the figure $X$ that satisfies

$$
X \oplus B=C .
$$

This is the inverse problem of the Minkowski sum.

The inverse problem can be partially solved by another operation called the Minkowski difference. The Minkowski difference of two figures $C$ and $B$, denoted $C \ominus B$, is defined as

$$
C \ominus B=\bigcap_{b \in B}\{c-b \mid c \in C\} .
$$

For the figures $B$ and $C$ in Fig. 1(b) and (c), respectively, $C \ominus B$ coincides with the figure in Fig. 1(a), and hence in this particular case, the solution of eq. (2) is given by $X=C \ominus B$.

However, the Minkowski difference does not give the complete solution of eq. (2). Actually the solution of eq. (2) is not necessarily unique. For example, let $A^{\prime}$ be the figure in Fig. 1 (e). Then, we get $A^{\prime} \oplus B=C$, and hence $X=A^{\prime}$ is also a solution of eq. (2). Furthermore, eq. (2) does not necessarily admit a solution. 


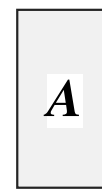

(a)

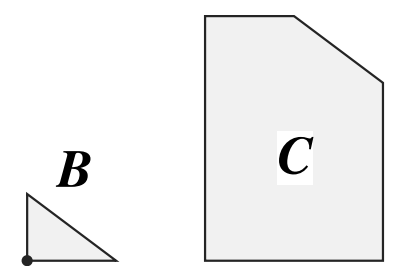

(b) (c)

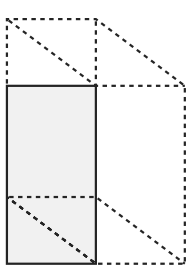

(d)

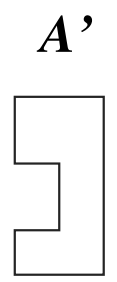

(e)

Fig. 1. Minkowski sum of two polygons.

\section{Angle-Parametric Polygonal Curves}

While the conventional Minkowski sum is defined on polygons, we consider a class of closed polygonal curves, a typical example of which is the boundary curve of a polygon. Let $\mathrm{P}$ be a closed polygonal curve with $n$ vertices $v_{0}, v_{1}, \cdots, v_{n-1}, v_{n}=$ $v_{0}$ and $n$ edges $e_{1}, e_{2}, \cdots, e_{n}$ such that edge $e_{i}$ is a line segment connecting $v_{i-1}$ to $v_{i}$. We consider that the edge $e_{i}$ has the direction from $v_{i-1}$ to $v_{i}$.

Examples of closed polygonal curves are shown in Fig. 2. The polygonal curve in Fig. 2(a) is considered the boundary of a polygon surrounding counterclockwise, while the one in Fig. 2(b) has a self-intersection and hence does not correspond to a polygon in an ordinary sense.

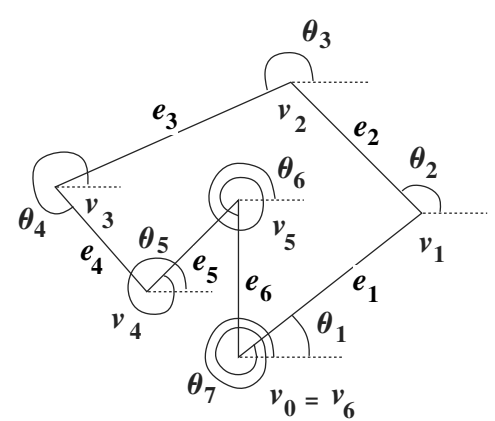

(a)

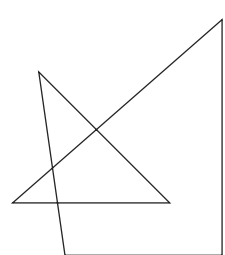

(b)

Fig. 2. Polygon.

For our convenience of the notation, let us assume that we travel along $\mathrm{P}$ around in either direction endlessly, and name the vertices and the edges accordingly, i.e., $v_{i+k n}=v_{i}$ and $e_{i+k n}=e_{i}$ for $k= \pm 1, \pm 2, \pm 3, \cdots$.

For each $i=0, \pm 1, \pm 2, \cdots$, let $\phi_{i}$ be the angle required when we turn from the direction of $e_{i}$ to the direction of $e_{i+1}$ counterclockwise. We call $\phi_{i}$ the left-turn 
angle at $v_{i}$. Since we measure the angle counterclockwise, we have $0<\phi_{i}<\pi$ if $\mathrm{P}$ bends to the left at $v_{i}$ as in Fig. 3(a), while we have $\pi<\phi_{i}<2 \pi$ if $\mathrm{P}$ bends to the right at $v_{i}$ as in Fig. 3. (b). We call the former vertex a convex vertex and the latter vertex a reflex vertex.

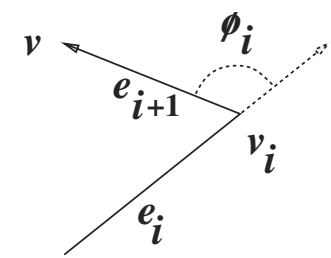

(a)

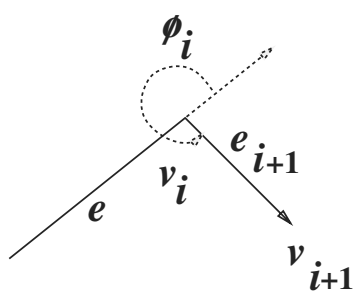

(b)

Fig. 3. Left-turn angle

Let us choose and fix an arbitrary edge, say $e_{s}$, and let $\theta_{s}$ be the angle of $e_{s}$ with respect to the positive $x$ direction measured counterclockwise with the convention that $0 \leq \theta_{s}<2 \pi$. Next, for each $i=0, \pm 1, \pm 2, \cdots$, we define

$$
\theta_{i}= \begin{cases}\theta_{s}+\phi_{s}+\phi_{s+1}+\cdots+\phi_{i-1} & \text { if } i>s \\ \theta_{s}-\phi_{s-1}-\phi_{s-2}-\cdots-\phi_{i} & \text { if } i<s\end{cases}
$$

In other words, $\theta_{i}$ basically represents the direction of the edge $e_{i}$, but the ambiguity of a multiple of $2 \pi$ is resolved by accumulating the left-turn angles starting with $\theta_{s}$, as shown in Fig. 2(a).

For $\theta \in \mathbf{R}$, let $f(\theta)$ be the set of points defined by

$$
f(\theta)= \begin{cases}\left\{v_{i}\right\} & \text { if } \theta_{i}<\theta<\theta_{i+1} \\ \left\{t v_{i-1}+(1-t) v_{i} \mid 0<t<1\right\} & \text { if } \theta=\theta_{i}\end{cases}
$$

That is, if $\theta=\theta_{i}, f(\theta)$ represents the set of points on the open line segment $e_{i}$, and otherwise $f(\theta)$ represents the set of a single point representing the vertex $v_{i}$ satisfying $\theta_{i}<\theta<\theta_{i+1}$. We call $f(\theta)$ the angle-parametric polygonal curve.

The function $f(\theta)$ is periodic. If $\mathrm{P}$ is the counterclockwise boundary of a convex polygon, the total left turn we make while we travel along $\mathrm{P}$ around is equal to $2 \pi$, and hence

$$
f(\theta)=f(\theta+2 \pi)
$$

On the other hand, if $\mathrm{P}$ is the counterclockwise boundary of a polygon with $k$ reflex vertices, the total left turn we make while we travel along $\mathrm{P}$ around is equal to $(k+1) 2 \pi$, and hence we get

$$
f(\theta)=f(\theta+(k+1) 2 \pi) .
$$


We call the smallest positive integer $m$ satisfying $f(\theta)=f(\theta+2 m \pi)$ the winding number of the polygonal curve.

If $\mathrm{P}$ is the boundary of a convex polygon, its angle-parametric representation $f(\theta)$ is unique. However, for a general polygonal curve $\mathrm{P}$, the angle parametric representation $f(\theta)$ depends on the choice of the starting edge $e_{s}$. For example, for the polygonal curve in Fig. 2(a), if we choose $e_{1}, e_{2}, e_{3}$, or $e_{4}$ as $e_{s}$, we get the same angle-parametric representation, while if we choose $e_{5}$ or $e_{6}$ as $e_{s}$, the corresponding angle-parametric representation is different from the former. In general, two edges $e_{i}$ and $e_{i+k}(k>0)$ give the same angle-parametric representation if and only if we do not face the positive $x$ direction when we turn from the direction of $e_{i}$ to the direction of $e_{i+k}$ counterclockwise. Hence, if the angleparametric representation $f(\theta)$ of the closed polygonal curve $\mathrm{P}$ has the winding number $m$, there are $m$ different angle-parametric representations for $\mathrm{P}$. We consider them different angle-parametric polygonal curves. In order to distinguish between the closed polygonal curve $\mathrm{P}$ and its angle-parametric representation $f(\theta)$, we call $\mathrm{P}$ the geometric polygonal curve.

In what follows, $f(\theta)$ represents the set of points at the particular value $\theta$ of the parameter, and the function itself is represented by $f$ or $\{f(\theta)\}$.

Let $\Pi^{+}$be the set of all angle-parametric polygonal curves. Next, we introduce a binary operation to $\Pi^{+}$, which corresponds the Minkowski sum.

\section{New Minkowski Sum}

For $\{f(\theta)\},\{g(\theta)\} \in \Pi^{+}$, we introduce a new operation $\oplus$ in the following way. First, for each value of the parameter $\theta$, we define

$$
f(\theta) \oplus g(\theta)=\{a+b \mid a \in f(\theta), b \in g(\theta)\},
$$

where $a+b$ denotes the sum of the radial vectors $a$ and $b$. That is, if both $f(\theta)$ and $g(\theta)$ consist of a single point, $f(\theta) \oplus g(\theta)$ also consists of a single point; if one of $f(\theta)$ and $g(\theta)$ consists of a point and the other is an edge, $f(\theta) \oplus g(\theta)$ is the line segment obtained by translating the edge by the radial vector representing the point; if both $f(\theta)$ and $g(\theta)$ are edges, $f(\theta) \oplus g(\theta)$ is an open line segment connecting the sum of the start points to the sum of the end points.

For the convenience in the subsequent discussion, we introduce the following notation. For each value of $\theta$, we consider $f(\theta)$ the line segment; that is, if $f(\theta)$ is a point, we consider it the line segment with length 0 . Let $f^{\mathrm{s}}(\theta)$ and $f^{\mathrm{e}}(\theta)$ be the start point and the end point of the line segment $f(\theta)$. Hence, if $f(\theta)$ is a point, we have $f^{\mathrm{s}}(\theta)=f^{\mathrm{e}}(\theta)$.

Next, we define $\{f(\theta)\} \oplus\{g(\theta)\}$ as

$$
\{f(\theta)\} \oplus\{g(\theta)\}=\{f(\theta) \oplus g(\theta)\},
$$

that is, $\{f(\theta)\} \oplus\{g(\theta)\}$ is a function of the angle parameter $\theta$, and its value at each $\theta$ is specified by eq. (8). As $\theta$ increases, almost everywhere $\{f(\theta)\} \oplus\{g(\theta)\}$ stays a constant singleton representing a vertex, and at some discrete values of 
$\theta$ it represents an edge connecting the vertex immediately before and the vertex immediately after. Moreover, $\{f(\theta)\} \oplus\{g(\theta)\}$ is periodic. Indeed its winding number is the least common multiple of the winding numbers of $\{f(\theta)\}$ and $\{g(\theta)\}$. Hence, $\{f(\theta)\} \oplus\{g(\theta)\}$ also belongs to $\Pi^{+}$. We call $\{f(\theta)\} \oplus\{g(\theta)\}$ the Minkowski sum of $\{f(\theta)\}$ and $\{g(\theta)\}$.

For example, let $\{f(\theta)\}$ and $\{g(\theta)\}$ be the angle-parametric representations of the polygon $A$ in Fig. 1(a) and the polygon $B$ in Fig. 1(b), respectively. Then, $\{f(\theta)\} \oplus\{g(\theta)\}$ coincides with the angle-parametric representation of the polygon $C$ in Fig. 1 (c).

Fig. 4 shows another example. The polygonal curves in Fig. 4 (a) and (b) both have winding number 2 , and hence they have two different angle-parametric representations. Let $f_{1}$ and $f_{2}$ be the two angle-parametric representations of the polygonal curve in Fig. 4(a), and $g_{1}$ and $g_{2}$ be the two angle-parametric representations of the polygonal curve in Fig. 4(b). Since $f_{1} \oplus g_{1}=f_{2} \oplus g_{2}$ and $f_{1} \oplus g_{2}=f_{2} \oplus g_{1}$, there are essentially two different Minkowski sums. They are shown in Fig. 4(c) and (d).

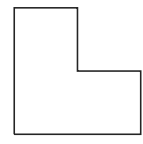

(a)

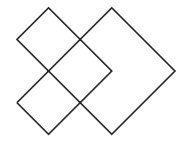

(b)

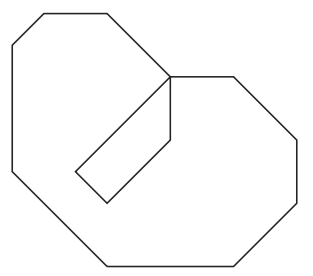

(c)

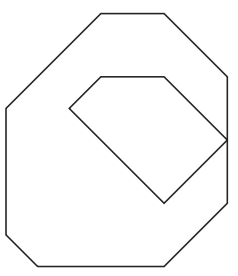

(d)

Fig. 4. Minkowski sums of two polygons with winding number 2 .

In this case also we can consider the polygons bounded by the outermost part of the polygonal curves. The conventional Minkowski sum of the polygons in Fig. 4(a) and (b) corresponds to the union of the polygons in Fig. 4l(c) and (d).

\section{Extension to the Invertible World}

As shown in eqs. (8) and (9), the Minkowski sum is defined in terms of the sum of radial vectors. Consequently, the algebraic structure of the vector space is inherited in $\Pi^{+}$. In particular, the following hold for any $f, g, h \in \Pi^{+}$.

Property 1. $f \oplus g=g \oplus f$ (commutativity).

Property 2. $(f \oplus g) \oplus h=f \oplus(g \oplus h)$ (associativity).

Property 3. $f \oplus g=f \oplus h$ implies $g=h$ (injectivity). 
There is a big theorem in algebra which says that, if Properties 1,2 and 3 are satisfied, the set $\Pi^{+}$can be augmented in such a way that all the elements have their inverses in it [2]. A well-known example is the extension of the set of natural numbers with the multiplication operation to the set of positive rational numbers.

More precisely, we can augment $\Pi^{+}$to a larger set $\Pi$ in the following way.

Let $\Pi^{+} \times \Pi^{+}$be the set of all ordered pairs of the elements of $\Pi^{+}$. For any $\left(f_{1}, f_{2}\right),\left(g_{1}, g_{2}\right) \in \Pi^{+} \times \Pi^{+}$, we define $\left(f_{1}, f_{2}\right) \oplus\left(g_{1}, g_{2}\right)$ by

$$
\left(f_{1}, f_{2}\right) \oplus\left(g_{1}, g_{2}\right)=\left(f_{1} \oplus g_{1}, f_{2} \oplus g_{2}\right) .
$$

[In the case of the natural numbers, eq. (10) corresponds to $\left(f_{1} / f_{2}\right) \cdot\left(g_{1} / g_{2}\right)=$ $\left.\left(f_{1} \cdot g_{1}\right) /\left(f_{2} \cdot g_{2}\right) \cdot\right]$

Next, we write $\left(f_{1}, f_{2}\right) \sim\left(g_{1}, g_{2}\right)$ if and only if $f_{1} \oplus g_{2}=f_{2} \oplus g_{1}$. [In the case of the natural nubmers, $f_{1} / f_{2} \sim g_{1} / g_{2}$ if and only if $f_{1} \cdot g_{2}=f_{2} \cdot g_{1}$.] Then, the binary relation $\sim$ is an equivalence relation in $\Pi^{+} \times \Pi^{+}$. Finally, we define $\Pi$ as the set all equivalence classes, that is, the quotient of $\Pi^{+} \times \Pi^{+}$by $\sim$ :

$$
\Pi=\Pi^{+} \times \Pi^{+} / \sim .
$$

[In the case of the natural numbers, $f_{1} / f_{2}$ is considered a rational number, and $f_{1} / f_{2}$ and $g_{1} / g_{2}$ are considered identical if $f_{1} / f_{2} \sim g_{1} / g_{2}$.]

Elements of our new world $\Pi$ can be considered more concretely in the following way. Let $e(\theta)$ be the origin for any $\theta$. Then $\{e(\theta)\}$ (or $e$ for short) is considered the "angle-parametric closed curve" consisting of the single point at the origin. Hence $e^{\mathrm{s}}(\theta)=0$ and $e^{\mathrm{e}}(\theta)=0$ for any $\theta$. e acts as the unit in $\Pi$, because for any $f \in \Pi^{+}$, we get $f(\theta) \oplus e(\theta)$ is the line segment from $f^{\mathrm{s}}(\theta)+e^{\mathrm{s}}(\theta)=f^{\mathrm{s}}(\theta)$ to $f^{\mathrm{e}}(\theta)+e^{\mathrm{e}}(\theta)=f^{\mathrm{e}}(\theta)$.

For any $f \in \Pi^{+}$, the inverse $f^{-1}$ of $f$ in $\Pi$ is considered the angle-parametric closed curve for which the line segment $f^{-1}(\theta)$ at each value of $\theta$ starts at $-f^{\mathrm{s}}(\theta)$ and ends at $-f^{\mathrm{e}}(\theta)$. In other words, we get $\{f(\theta)\}^{-1}=\{-f(\theta)\}$. More generally, for any $f, g \in \Pi^{+}$, the equivalence class containing $(f, g)$ can be considered the angle-parametric closed curve whose value at each $\theta$ is the line segment from $f^{\mathrm{s}}(\theta)-g^{\mathrm{s}}(\theta)$ to $f^{\mathrm{e}}(\theta)-g^{\mathrm{e}}(\theta)$. Namely, the equivalence class containing $(f, g)$ can be written $\{f(\theta)-g(\theta)\}$.

The algebraic structure of the extension from $\Pi^{+}$to $\Pi$ is the same as that of the extension from the natural numbers to the positive rationals. Similar extension was also applied when the world of functions was augmented to the world of hyperfunctions [1]. From that analogy, we call elements of $\Pi-\Pi^{+}$ hypercurves or hyperpolygons.

\section{Interpretation of Hyper Curves}

Let us consider a numerically controlled polish machine as shown in Fig. 5 . In this figure, $A$ is a rail, $B$ is a support plate, $C$ is an arm, $D$ is a polish disc, and $E, F, G$ constitute the material to be polished. The upper part of Fig. 5 is the 


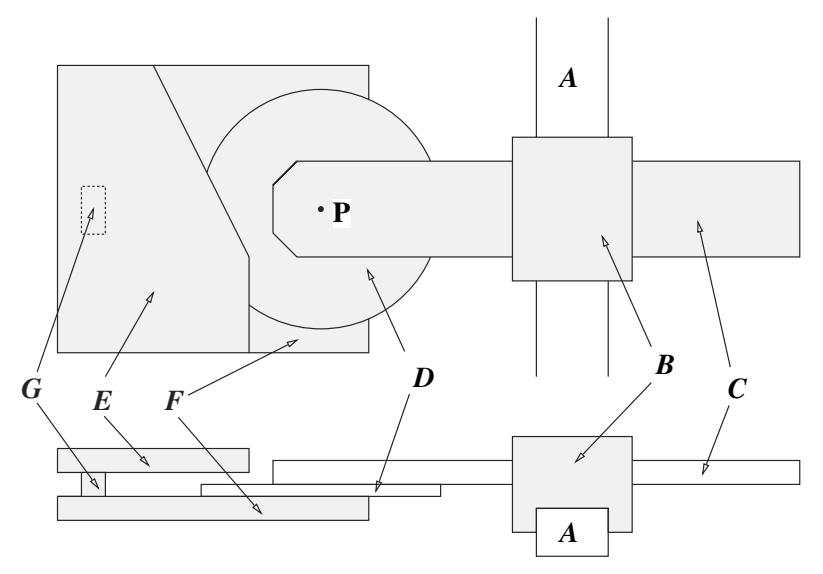

Fig. 5. Numerically controlled polish machine.

top view (the projection on the $x y$ plane) while the lower part is the side view (the projection on the $x z$ plane).

The rail $A$ is fixed to the outside world, on which the support plate $B$ slides in the $y$ direction. The arm $C$ is held by $B$ and can slide in the $x$ direction. The polish disc is attached to the arm at $\mathrm{P}$ and can rotate around $\mathrm{P}$. The material consists of mutually parallel two plates $E$ and $F$, and they are connected by a small $\operatorname{rod} G$. The material is fixed to the outside world, and the upper surface of the plate $F$ is to be polished by $D$.

Note that the arm $C$ cannot enter the material region $E$, because $C$ and $E$ are on the same $z$ level, as shown in the side view of Fig. 5 . If the arm $C$ enters the material region $E$, they collide and broke each other. Hence the arm $C$ can move only outside $E$.

We are interested in which part of the surface of $E$ can be polished by this machine. To answer this question, we usually apply the Minkowski algebra in the following way.

Let $R(C)$ represent the polygon obtained when we rotate the polygon $C$ by 180 degrees around the point $\mathrm{P}$, and let $\left\{f_{R(C)}(\theta)\right\},\left\{f_{D}(\theta)\right\}$ and $\left\{f_{E}(\theta)\right\}$ be the angle-parametric polygonal curves representing the shapes of $R(C), D$ and $E$, respectively. We assume that the point $\mathrm{P}$ is chosen as the origin of the coordinate system to represent $f_{R(C)}$ and $f_{D}$. Then the Minkowski sum $g=f_{E} \oplus f_{R(C)}$ represents the region which the reference point $\mathrm{P}$ of the arm $C$ cannot enter.

Next, let $\{h(\theta)\}$ be the Minkowski difference; $h$ is expressed by

$$
h=g \oplus f_{D}^{-1}=\left(f_{E} \oplus f_{R(C)}\right) \oplus f_{D}^{-1} .
$$

The region represented by $h$ corresponds to the region which the polish disc cannot reach. Hence, the intersection of this region with the material region $E$ is the region that cannot be polished by this machine. This is the conventional 
way of finding the unpolishable region of the material surface (although we used our new notations). Note that the above computation can be done only after we are given the shape of the material $E$.

In our new algebra, on the other hand, we can compute the Minkowski sums in any order, because the algebra forms a group and the uniqueness of the final result is guaranteed. Now, let us rewrite eq. (12) into

$$
h=\left(f_{R(C)} \oplus f_{D}^{-1}\right) \oplus f_{E} .
$$

Let $\{w(\theta)\}$ denote the first part of the right-hand side of the equation: $w=$ $f_{R(C)} \oplus f_{D}^{-1} \cdot w$ is a hypercurve.

If we compute according to eq. (12), the intermediate result $g$ and the final result $h$ are both in $\Pi^{+}$. On the other hand, if we compute according to eq. (13), the intermediate result $w$ goes out from $\Pi^{+}$, and hence the computation becomes invalid in the conventional algebra.

This difference is important, because $w$ can be computed even if we are not given the actual shape of the material. Actually, $w$ depends only on the polish machine, and hence we can interpret that the hypercurve $w$ represents the ability of the polish machine.

This example shows the following two important points. First, the hypercurve has its own physical meaning, just as the "ability of the polish machine" in the above example. Secondly, the hypercurve can save the computational cost, because we need to compute the intermediate result $w$ only once, and can apply it to any material shape, whereas in the conventional method we have to compute the intermediate result $g$ every time a new material $E$ is given.

\section{Concluding Remarks}

We have reformulated the Minkowski algebra in such a way that the new algebra forms a group and consequently the sum and its inverse can always be taken freely. In this new algebra, the conventional polygonal curves are extended to more general geometric objects, which we name the "hypercurves". We also gave a physical interpretation of the hypercurve, and discussed the practical use of this new concept.

There still remains many related problems to be solved. They include (i) efficient algorithms for this algebra, (ii) optimal representation of a given polygonal region by an angle-parametric polygonal curve, (iii) physical interpretation of hypercurves generated by nonconvex curves, and (iv) extension to higher dimensions.

Acknowledgements. This work is supported by the Grant-in-Aid for Scientific Research of the Japanese Ministry of Education, Science, Sports and Culture. 


\section{References}

1. H. Bekker and J.B.T.M. Roerdink: An efficient algorithm to calculate the Minkowski sum of convex 3D polyhedra. Lecture Notes in Computer Science 2073, pp. 619-628, 2001.

2. N. Bourbaki: Éléments de Mathématique, Algébre 1. Hermann, Paris, 1964.

3. P. K. Ghosh: An algebra of polygons through the notion of negative shapes. CVGIP: Image Understanding, vol. 54 (1991), pp. 119-144.

4. P. K. Ghosh: Vision, geometry, and Minkowski operators. Contemporary Math., vol. 119 (1991), pp. 63-83.

5. P.K. Ghosh and R.M. Haralick: Mathematical morphological operations of boundary-represented geometric objects. J. Math. Imaging and Vision, vol. 6 (1996), pp. 199-222.

6. L. J. Guibas, L. Ramshaw and J. Stolfi: A kinetic framework for computational geometry. Proc. 24th IEEE Symp. Foundation of Computer Sciences, (1983), pp. $100-111$.

7. S. Har-Peled, T. M. Chan, B. Aronov, D. Halperin and J. Snoeyink: The complexity of a single face of a Minkowski sum. Proc. 7th Canadian Conf. Comput. Geom., (1995), pp. 91-96.

8. A. Hermandez-Barrera: Computing the Minkowski sum of monotone polygons. IEICE Transactions on Information and Systems, vol. E80-D (1997) pp. 218-222.

9. M.-S. Kim and K. Sugihara: Minkowski sums of axis-parallel surfaces of revolution defined by slope-monotone closed curves. IEICE Transactions on Information and Systems, vol. E84-D (2001), pp. 1540-1547.

10. D. Leven and M. Sharir: Planning a purely translational motion for a convex object in two-dimensional space using generalized Voronoi diagram. Discrete and Comput. Geom., vol. 2 (1987), pp. 9-31.

11. J. Mikusinski: Operational Calculus. Pergamon Press, London, 1957.

12. D. Mount and R. Silverman: Combinatorial and computational aspects of Minkowski decompositions. Contemporary Math., vol. 119 (1991), pp. 107-124.

13. G. D. Ramkumar: An algorithm to compute the Minkowski sum outer-face of two simple polygons. Proc. 12th ACM Symp. Comput. Geom., (1996), pp. 234-241.

14. K. Sugihara, T. Imai and T. Hataguchi: An algebra for slope-monotone closed curves. Int. J. Shape Modeling, vol. 3 (1997), pp. 167-183. 